\title{
Editorial: Forschungskulturelle Kontexte
}

\section{Timo Storck}

Angenommen: 14. April 2021

(C) Springer Medizin Verlag GmbH, ein Teil von Springer Nature 2021

\begin{abstract}
„Man kann sich des Eindrucks nicht erwehren, daß die Menschen gemeinhin mit falschen Maßstäben messen ...“ - so beginnt Freud seine Arbeit „Das Unbehagen in der Kultur“ (Freud 1930a, S. 421). Mit den falschen Maßstäben bezieht er sich hier auf „Macht, Erfolg und Reichtum“ gegenüber den „wahren Werte[n] des Lebens“ (ebd.), und dieser Einstieg dient ihm als Einleitung zur Erwähnung des ,ausgezeichneten Mannes“ (gemeint ist: Romain Rolland), der ihm vom „ozeanischen Gefühl“" geschrieben hat, einem Gefühl der Verbundenheit mit der Umwelt, einem „gutartigen“ Verlust der Ich-Grenzen. Erste Szenen und erste Sätze in einer solchen Abhandlung wie auch in klinischen Behandlungen sind für eine psychoanalytische Betrachtung von besonderer Bedeutung. Hat jedwede Kultur etwas mit Vereinigung zu tun? Gilt das auch für die unterschiedlichen und reichhaltigen Themenfelder psychoanalytischer Kulturforschung und Forschungskultur?
\end{abstract}

\section{Aneignung und Befremdung}

Freuds Kulturtheorie unterstreicht deshalb das Unbehagen, weil menschliche Kulturleistungen einer Einschränkung des Lustprinzips bedürfen. Handeln unter Beachtung sozialer Realität, bezogene Intimität, Zusammenarbeit und überhaupt: (sekundär prozesshaftes) Denken sind für Freud Kulturleistungen, aber eben auch Kompromissbildungen, die das egoistische Interesse des Menschen beinhalten und kanalisieren. Das ist als Grundlage einer Kulturtheorie gleichwohl nicht das pessimistische Bild, als das es manchmal aufgenommen worden ist. Es geht um eine konstitutive Spannung zwischen Individuum und Gesellschaft und damit um die Aufgabe eines sozial

Prof. Dr. Dipl.-Psych. T. Storck $(\varangle)$

Klinische Psychologie und Psychotherapie, Psychologische Hochschule Berlin, Berlin, Deutschland E-Mail: t.storck@psychologische-hochschule.de 
vermittelten, verantwortungsvollen Handelns, das als solches eben nicht „naturgegeben“" ist, sondern geformt und mühsam errungen. Kultur selbst ist das Resultat psychischer wie sozialer Bearbeitung.

Nun ist jeder Kultur-Begriff dann problematisch, wenn er einem Kulturalismus Vorschub leistet. Indem von „Kultur“ gesprochen wird, sollte nicht verdeckt werden, dass damit etwas immer schon Spannungsreiches adressiert ist, und eine Sensibilisierung dafür gegeben sein, wann Zuschreibungen ausgrenzend, stereotypisierend oder anderweitig statisch sind. Wenn es um Kultur geht, ist das Spannungsfeld zwischen „Eigenem“" und „Fremdem“ angesprochen, wobei das Entscheidende ist, sich des Fremden im Selbst und des Geteilten in der interpersonellen Begegnung zu vergewissern. Selbst noch „Eigenes“ und „Fremdes“ können nicht als statische Kategorien gebraucht werden. Auch Kultur ist immer unheimlich statt behaglich, eine Art Heimstätte, in der das menschliche Ich doch nie so ganz Herr im eigenen KulturHause ist. Homo culturalis zu sein, bedeutet dann, die konstant dynamische Herausforderung einer Aneignung statt Vereinnahmung, eines Anerkennens von Differenz und (Selbst-)Befremdung statt eines statischen ,othering“ im Sinne der stereotypisierenden Zuordnung zu einem isolierten Anderssein. Dabei sollte nicht übergangen werden, dass Praktiken der Aneignung, Integration, Partizipation ebenso Ausdruck einer ausgeübten „Definitionsmacht“ von Kultur sein können wie die stereotypisierende Ausgrenzung.

Auf der Basis solcher Perspektiven auf „kulturelle Kontexte“ lässt sich aus Sicht der Psychoanalyse einiges sagen, zum Beispiel die analytische Sozialpsychologie oder die Kunstpsychoanalyse u. a. zeigen das, aber auch die zunehmende psychoanalytische Auseinandersetzung mit Diversität und der eigenen Denk-Kultur im Hinblick auf Geschlecht und Geschlechtsidentität, sexuelle Orientierung, Herkunft und Ethnie, Hautfarbe oder sozioökonomische Stellung und Rollenbeziehungen.

\section{Das Unbehagen in der Psychotherapieforschungskultur}

Die Beiträge des vorliegenden Schwerpunkthefts greifen einen spezifischen Aspekt von „Kultur“ heraus, indem sie sich der Forschungskultur und der Stellung der Psychoanalyse darin widmen. Eine wechselseitige Skepsis zwischen wissenschaftlicher Forschung und klinischer Psychoanalyse durchzieht die Geschichte, was sich bereits in Freuds (1925e) Bemerkungen über die Widerstände gegen die Psychoanalyse zeigt. Ob es dabei so nützlich gewesen ist, den Kritikern der Psychoanalyse psychodynamisch relevante Motive vorzuhalten, mag dahingestellt sein, aber jedenfalls hat die Psychoanalyse von Beginn an das Bild einer Sonderstellung im Feld von Wissenschaft und Forschung für sich beansprucht. Sie ist zudem nicht nur immer wieder kritisiert worden, sondern hat sich selbst den Platz einer kritischen Instanz gegeben.

Das mag man nun mit dem Bild des ,Stachels“ umschreiben, als der die Psychoanalyse sich für die (übrige) Wissenschaft gelegentlich sieht, die kritische Instanz, die als solche immer wieder an einen Selbstreflexionsbedarf gemahnt, oder mit dem Bild des „Elfenbeinturms“, in dem die Psychoanalyse sich zurückgezogen hat. Zumindest in christlich-religiöser Betrachtung bezieht sich der Elfenbeinturm im 
Übrigen ja nicht nur auf die Abgeschiedenheit, sondern vor allem auf die Reinheit, Unberührtheit, Jungfräulichkeit. Reinheitsfantasien sind hier gleichwohl problematisch, denn sie führen „wissenschaftskulturell“ in eine statische, entwicklungslose Position.

Die Vorgehensweise und „Kultur“ der „akademischen“ Welt sind in sich natürlich sehr viel heterogener, als es in dieser Gegenüberstellung scheint, und die Erkenntnishaltungen von Psychoanalyse und ,den anderen“ sind oft als einander widerständig, gar inkommensurabel aufgefasst worden, zumindest und insbesondere in Medizin und Psychologie. Das findet im Verhältnis der quantitativ-empirischen Psychotherapieforschung und der klinischen Psychoanalyse einen besonders deutlichen Ausdruck. In „Das Unbehagen in der Kultur“ weist Freud (1930a, S. 470) auch auf die Wurzeln der moralischen, kulturstiftenden Prämisse, man solle seinen Nächsten lieben wie sich selbst, hin: Aus welchen Gründen, so Freud, wenn nicht aus solchen der basalen Aggression gegenüber seinem Nächsten, sollte diese kulturelle Forderung überhaupt erhoben werden? Insbesondere in der genannten Art der Psychotherapieforschung mit ihrer langen Zeit von wechselseitiger Skepsis bis Entwertung zwischen den psychotherapeutischen Behandlungsverfahren (insbesondere kognitiv-behavioral und analytisch) und einem „Pferderennen“ um die Wirksamkeit geprägten „Kultur“, mag man den Eindruck haben, die Forderung „Liebe das Verfahren deines Nächsten wie dein eigenes" sei vollkommen auf ihr Grundgerüst von vermeintlich selbstbehauptender Aggression und Destruktivität reduziert worden.

Versteht man die verschiedenen psychotherapeutischen Verfahren nun als etwas, in dem eine jeweilige „Kultur“ vertreten wird, dann wird auch hier die Frage nach dem Einbezug des Fremden in die eigene „Kultur“ von Interesse. Wie blickt man auf das andere, Fremde?

Zwei Probleme werden durch die Einführung des Kultur-Begriffs in die Betrachtung des Verhältnisses von Psychoanalyse und „,akademischer“ Forschung gut sichtbar:

1. Die Gefahr eines „othering“, also des stereotypisierenden Blicks auf das ganz andere, zur (letztlich wenig stabilen) Sicherung des Gefühls von eigener „Identität“ durch Ab- und Ausgrenzung,

2. die Gefahr einer schlichten Vereinnahmung (statt vermittelter Aneignung) von etwas, das seine Bedeutung in einem anderen Kontext hat.

Der erste Punkt wäre berührt, wenn etwa ein psychotherapeutisches Verfahren auf ein anderes blickt, indem diesem bestimmte Haltungen oder Eigenschaften zugeschrieben werden, die weder hinterfragt noch erweitert werden, zum Beispiel: „Psychoanalytiker sind immer möglichst kühl und beantworten jede Frage mit einer Gegenfrage“ oder „Verhaltenstherapeutinnen malen immer alles auf Flipcharts“. Vor diesem Hintergrund würde dann das jeweils Eigene in einer radikalen Differenz zu etwas ganz anderem bestimmt. Der zweite Punkt ist dann relevant, wenn einer Art des Eklektizismus in der Psychotherapie gefolgt wird, in der es keine Beachtung findet, dass einzelne Konzepte in einem konzeptuellen Zusammenhang mit anderen oder einzelne Interventionen in einer Behandlungsplanung stehen, zum Beispiel, wenn ohne weitere Vorbereitung in einer analytischen Behandlung eine In-vivo- 
Exposition eingeschaltet oder in einer kognitiv-behavioralen Therapie plötzlich die Deutung eines Übertragungswiderstands gegeben würde.

Gemeinsam ist beiden Gefahren im psychotherapeutischen Kulturenvergleich, dass die Spannung, welche Kultur(en) auszeichnet, zugunsten eines ozeanischen Gefühls von spannungsloser Einheit ohne Differenz negiert wird. Zu beachten ist dies in jedem Versuch der „Psychotherapie-Integration“ (Sell und Benecke 2020). Gemäß dem oben skizzierten Kulturbegriff, der mit Aneignung und Sich-Befremden sowie der Reflexion der Bezüge beider zueinander zu tun hat, geht es darum, Differenz weder zu überhöhen noch aufzulösen oder zu negieren.

Die drei Beiträge des Schwerpunkts „Forschungskulturelle Kontexte“ widmen sich dieser Aufgabe: Maren Kellermann betrachtet Publikationskulturen in der psychosomatischen Medizin im Wandel der vergangenen 50 Jahre. Christian Sell untersucht Methodenkulturen, das heißt hier: die Möglichkeiten einer „Mixed-methods“Psychotherapieforschung, in die sich die Psychoanalyse mit ihren Methoden, aber auch ihren Fragestellungen einbringt. Timo Storck, Jana Volkert, Felix Brauner und Christian Sell widmen sich den klinischen Kulturen der Psychotherapie-Verfahren und machen einen Vorschlag zur Untersuchung therapeutischer Arbeitsmodelle im Schulenvergleich.

Den drei Beiträgen zur „Forschungskulturforschung“ ist gemeinsam, dass sie einen genuinen psychoanalytischen Beitrag zur Forschung liefern, in einer Weise, die nicht bloß für die Psychoanalyse relevant ist, sondern einer - spannungsreich bleibenden - Auseinandersetzung unterschiedlicher Zugangsweisen zu Wissenschaft und klinischer Praxis in den jeweiligen Kulturen gegenüber offen bleibt, sodass Forschungsfragen in vertiefter Weise und unter Einbezug psychodynamischen Denkens adressiert werden können.

Freuds „Unbehagen in der Kultur“" (1930a, S. 506) endet übrigens mit den Worten: „Aber wer kann den Erfolg und Ausgang voraussehen?“ ...

Interessenkonflikt T. Storck gibt an, dass kein Interessenkonflikt besteht.

\section{Literatur}

Freud S (1925e) Die Widerstände gegen die Psychoanalyse. GW, Bd. XIV, S 97-110

Freud S (1930a) Das Unbehagen in der Kultur. GW, Bd. XIV, S 419-506

Sell C, Benecke C (2020) Methodenintegration in der psychodynamischen Psychotherapie. Möglichkeiten, Grenzen und neue Möglichkeiten. PDP 19(2):185-200

Prof. Dr. Dipl.-Psych. Timo Storck Prof. Dr. phil., Psychoanalytiker (DPV/DGPT/IPA), Professor für Klinische Psychologie und Psychotherapie an der Psychologischen Hochschule Berlin und psychologischer Psychotherapeut (AP/TP). Aktuelle Buchpublikationen: Grundelemente psychodynamischen Denkens (bisher 6 Bände; Kohlhammer, $2018 \mathrm{ff}$.), Denken und Lernen (Kohlhammer, 2021; mit F. Billhardt), Wahrnehmung und Gedächtnis (Kohlhammer, 2021; mit F. Billhardt), Psychoanalyse nach Sigmund Freud (Kohlhammer, 2018). 